\title{
Cancer and Aging Research Group Chemo Toxicity Calculator
}

National Cancer Institute

\section{Source}

National Cancer Institute. Cancer and Aging Research Group Chemo Toxicity Calculator. NCI Thesaurus. Code C134827.

A clinical assessment tool designed to evaluate the risk for chemotherapy toxicity in geriatric cancer patients. The tool captures sociodemographics, tumor and treatment variables, laboratory test results, and geriatric assessment domains. 